\title{
On the Maximum and Minimum of Double Generalized Gamma Variates with Applications to the Performance of Free-space Optical Communication Systems
}

\author{
Hessa AlQuwaiee, Student Member, IEEE, Imran Shafique Ansari, Member, IEEE, \\ and Mohamed-Slim Alouini, Fellow, IEEE
}

\begin{abstract}
In this work, we derive the exact statistical characteristics of the maximum and the minimum of two modified ${ }^{1}$ double generalized gamma variates in closed-form in terms of Meijer's G-function, Fox's H-function, the extended generalized bivariate Meijer's G-function and $\mathbf{H}$-function in addition to simple closed-form asymptotic results in terms of elementary functions. Then, we rely on these new results to present the performance analysis of (i) a dual-branch free-space optical selection combining diversity and of (ii) a dual-hop free-space optical relay transmission system over double generalized gamma fading channels with the impact of pointing errors. In addition, we provide asymptotic results of the bit error rate of the two systems at high SNR regime. Computer-based Monte-Carlo simulations verify our new analytical results.
\end{abstract}

Index Terms-Free-space optical, double generalized Gamma, turbulence, pointing errors, dual-branch diversity, selection combining, dual-hop relay system, hybrid RF/FSO.

\section{INTRODUCTION}

Recently, radio frequency spectrum for wireless communications is facing a crunch. Research is driven to find solutions such as utilizing the spectrum more efficiently or using unregulated one such as the optical spectrum under which freespace optical (FSO) communications operates [5], [6]. FSO can complement or substitute radio frequency (RF) systems due to their higher bandwidth, cost effective, resistivity to

\footnotetext{
${ }^{1}$ Under the impact of pointing error (i.e. $I=I_{p} I_{a}$ where $I_{a}$ is the irradiance modeled as double generalized gamma random variable [1] and $I_{p}$ is the pointing error whose probability density function (PDF) can be found in [2, Eq. (10)]). The PDF of $I$ can be found in [3, Eq. (8)]

Copyright (c) 2015 IEEE. Personal use of this material is permitted. However, permission to use this material for any other purposes must be obtained from the IEEE by sending a request to pubs-permissions@ieee.org.

The research reported in this publication was supported by King Abdulaziz City of Sciences and Technology (KACST) under the Grant Agreement number AT-34-145 and by the King Abdullah University of Science and Technology (KAUST). Furthermore, it is important to note that our work in [4] is a special case of this paper.

H. Alquwaiee, and M.-S.Alouini are with King Abdullah University of Science and Technology (KAUST), Computer, Electrical and Mathematical Science and Engineering Division (CEMSE), Thuwal 23955-6900, Saudi Arabia (e-mail: hessa.alquwaiee, slim.alouini@kaust.edu.sa).

I. S. Ansari is currently with the Department of Electrical and Computer Engineering (ECEN), Texas A\&M University at Qatar (TAMUQ), Education City, Doha, Qatar. Please note when this work was initially submitted I. S. Ansari was with King Abdullah University of Science and Technology (KAUST), Computer, Electrical and Mathematical Science and Engineering Division (CEMSE), Thuwal 23955-6900, Saudi Arabia (email: imran.ansari@qatar.tamu.edu, imran.ansari@kaust.edu.sa).
}

interference and efficient employment time [7], [8]. This makes FSO applicable for broadband wireless technologies such as optical fiber backup, metropolitan area network, and last mile access [9]. On the other hand, FSO still have some challenges that can affect link range and system reliability such as atmospheric turbulence conditions (scintillation) that is comparable to the fading phenomena in the RF systems. Weather conditions can also affect signal strength as the air being the medium of the transmission. This can be solved by employing network redundancy along the transmission path. Moreover, thermal expansion, weak earth quakes, and dynamic wind loads can result in misalignment between the transmitter and the receiver introducing the pointing errors [2], [10]-[12].

Diversity, in which multiple copies of the same data is transmitted to the receiver such that each copy experiences different path/channel, has shown a great deal in mitigating fading effects in RF systems in addition to improving both performance and capacity [13]-[15]. For diversity combining techniques, selection combining (SC) is one of the most efficient and simple combining schemes. In SC, the receiver aims to process the branch with maximum signal-to-noise ratio (SNR). Diversity schemes in FSO systems was first introduced by Ibrahim [16] followed by great number of research instigating diversity over weak and strong turbulence conditions. Some investigated the spatial diversity and combining techniques of correlated and independent log-normal turbulence channels [17]-[20]. Also, spatial diversity was studied over K-distributed channels for different combining schemes in [21]. Moreover, SC over Gamma-Gamma under the impact of pointing errors has been investigated [4].

Relaying also has been an interesting area of research as it enhances the capacity of the system and provides energy efficient coverage. In this technique, a relay node is positioned in the way between transmitter and receiver to support direct data transmission. Multiple research works have been reported to analyze the relay system on both symmetric and asymmetric links (i.e. symmetric links when source-relay (S R) and relay-destination $(\mathrm{R} \sim \mathrm{D})$ links fall under the same fading model) [22]-[25]. Moreover, relaying over FSO system was introduced and then followed by several research work concerning symmetric multi-hop FSO systems over K-distributed, Gaussian and Gamma-Gamma turbulence channel [26]-[28]. On the other hand, several studies have been conducted to 
analyze asymmetric fixed and variable gain FSO relay systems as such integrating RF links in FSO systems. Specifically, assuming that RF and FSO links are, respectively, subject to Rayleigh and Gamma-Gamma or M-distributed turbulence channels [29]-[31]. Analyzing variable gain relay system analytically might not be tractable and mathematically not feasible. Throughout the years, the end-to-end SNR has been upper bounded by the minimum SNR among the sublinks [32] and harmonic mean of the each link SNR [33]. Few research work have been reported focusing on variable gain FSO relay systems over Gamma-Gamma with pointing errors [4], [34], [35].

Double generalized gamma channel model for free-space optical communication systems developed by [1] covers a very wide range of turbulence conditions and makes it generic to describe the FSO channel. Also, pointing errors was integrated to this model to better show the impact of pointing error impairments on the FSO system [3]. Hence, in this work, we study the dual-branch FSO selection combining and dualhop variable gain FSO relay operating on such channels with the impact of pointing errors to show diversity enhancement on the system performance and capacity. First, we express the statistical properties of the maximum of the minimum of double Generalized Gamma random variables under the impact of pointing errors in terms of $\mathrm{H}$-function and G-function. In particular, we find the cumulative distribution function (CDF), the probability density function (PDF), the moment generating function (MGF), and the moments in closed-form. We then use these results to evaluate performance measures such as the average bit error rate (BER) and the ergodic capacity (EC) of both FSO systems. Finally, we multiplex two independent FSO links with desired priority on the data rate of each links, thereby, utilizing the resources efficiently and effectively.

\section{Channel And System Models}

In this work, we consider a single FSO branch with two types of detection techniques, heterodyne and intensity modulation/direct detection (IM/DD). Data transmission is affected by path loss, atmospheric turbulence conditions, pointing errors, and additive white Gaussian noise (AWGN) that can be modeled as

$$
y_{i}=\eta I_{i} x+w_{i} \quad,
$$

where $\eta$ is the effective photoelectric conversion ratio, $w_{i}$ refers to the AWGN sample with power spectral density equals to $N_{0}$, and $I_{i}$ is the receiver irradiance that is defined as $I_{i}=I_{a, i} I_{p}$ where $I_{a, i}$ and $I_{p}$ reflect the turbulence-induced fading and the pointing error effect, respectively [36]. The end-to-end SNR is defined as $\gamma_{i}=\frac{I_{i}^{r_{i}}}{\mathbb{E}\left[I_{i}\right]^{r_{i}}} \mu_{r_{i}, i}$ where $r_{i}$ refers to the detection method (i.e. $r_{i}=1$ heterodyne detection and $\left.r_{i}=2 \mathrm{IM} / \mathrm{DD}\right), \mu_{r_{i}, i}$ is the average SNR defined in [37, Eq. (13)], and $\mathbb{E}[$.$] is the expectation operator. If we assume$ that the turbulence channel is modeled as double generalized Gamma under the assumption of pointing errors, then the CDF of $\gamma_{i}$ can be given as [37, Eq. (14)]

$$
\begin{aligned}
F_{\gamma_{i}}(\gamma) & =\frac{\xi_{i}^{2} \sigma_{i}^{\beta_{1 i}-\frac{1}{2}} \lambda_{i}^{\beta_{2 i}-\frac{1}{2}}(2 \pi)^{1-\frac{r_{i}\left(\lambda_{i}+\sigma_{i}\right)}{2}} r_{i}^{\beta_{1 i}+\beta_{2 i}-2}}{\alpha_{2 i} \lambda_{i} \Gamma\left(\beta_{1 i}\right) \Gamma\left(\beta_{2 i}\right)} \\
& \times \mathrm{G}_{r_{i}+1, u_{i}+1}^{u_{i}, 1}\left[C_{i}\left(\frac{\gamma}{\mu_{r_{i}, i}}\right)^{v_{i}} \mid \begin{array}{c|c}
1, \kappa_{3 i} \\
\kappa_{4 i}, 0
\end{array}\right],
\end{aligned}
$$

where $\xi_{i}=\frac{w_{e}}{2 \sigma_{s}}$ is the ratio between the equivalent beam width at the receiver and the pointing error displacement standard deviation (jitter), $\alpha_{x}, \beta_{x}$, and $\Omega_{x}$ are identified using the variance of the small and large scale fluctuations of the laser beam from [38, Eq. (18)-(20)] inserted in [1, Eq. (8a), (8b), (9)], $\lambda_{i}$ and $\sigma_{i}$ are positive integers such as $\frac{\lambda_{i}}{\sigma_{i}}=\frac{\alpha_{1 i}}{\alpha_{2 i_{i}}}, u_{i}=r_{i}\left(1+\lambda_{i}+\sigma_{i}\right)$, $v_{i}=\alpha_{2 i} \lambda_{i}, C_{i}=\left(\frac{A_{2, i} h_{i}^{v_{i}}}{r_{i}^{\left(\lambda_{i}+\sigma_{i}\right)}}\right)^{\alpha_{2 i}}, h_{i}=\frac{A_{1 i} B_{1 i}}{\left(1+\xi_{i}^{2} A_{2 i}^{1 / v_{i}}\right.}$, $A_{1 i}=\frac{\xi_{i}^{2} \sigma_{i}^{\beta_{1 i}-\frac{1}{2}} \lambda_{i}^{\beta_{2 i}-\frac{1}{2}}(2 \pi)^{1-\frac{\left(\lambda_{i}+\sigma_{i}\right.}{2}}}{\Gamma\left(\beta_{1 i}\right) \Gamma\left(\beta_{2 i}\right)}, A_{2 i}=\frac{\beta_{1 i}^{\sigma_{i}} \beta_{2 i}^{\lambda_{i}}}{\lambda_{i}^{\lambda_{i}} \sigma_{i}^{\sigma_{i}} \Omega_{1 i}^{\sigma_{i}} \Omega_{2 i}^{\lambda_{i}}}$, $B_{1 i}=\prod_{j=1}^{\sigma_{i}+\lambda_{i}} \Gamma\left(\frac{1}{v_{i}}+\kappa_{1 i, j}\right)$, where $\kappa_{i, j}$ is the $j$ th-term of $\kappa_{i}, \kappa_{1 i}=\Delta\left(\sigma_{i}: \beta_{1 i}\right), \Delta\left(\lambda_{i}: \beta_{2 i}\right), \kappa_{3 i}=\Delta\left(r_{i}: \frac{\xi_{i}^{2}+v_{i}}{v_{i}}\right)$ comprising of $r_{i}$ terms, and $\kappa_{4 i}=\left[\Delta\left(r_{i}: \kappa_{2 i}\right)\right]$ comprising of $u_{i}$ terms such that $\kappa_{2 i}=\xi_{i}^{2} / v_{i}, \kappa_{1 i}, \Delta(x: y) \triangleq \frac{y}{x}, \frac{y+1}{x} \ldots, \frac{y+x-1}{x}$ and $\left[\Delta\left(z: a_{m}\right)\right] \triangleq \Delta\left(z: a_{1}\right), \Delta\left(z: a_{2}\right) \ldots, \Delta\left(z: a_{m}\right)$.

By setting $\sigma_{i}=\lambda_{i}=\Omega_{i}=1, \beta_{1 i}=\alpha_{i}, \beta_{2 i}=\beta_{i}, h_{i}=$ $\frac{\xi_{i}^{2}}{\xi_{i}^{2}+1}, \xi_{i}>>1$ then $h_{i}=1, C_{i}=\left(\frac{\alpha_{i} \beta_{i}}{r_{i}^{2}}\right)^{r_{i}}, u_{i}=3 r_{i}, v_{i}=1$, $\kappa_{3 i}=\Delta\left(r_{i}: \xi_{i}^{2}+1\right), \kappa_{4 i}=\Delta\left(r_{i}: \xi_{i}^{2}\right), \Delta\left(r_{i}: \alpha_{i}\right), \Delta\left(r_{i}: \beta_{i}\right)$ we have the special case of Gamma-Gamma (GG) turbulence perturbed by pointing errors [4].

\section{Closed-Form Statistical Characteristics}

\section{A. Maximum of Two Variates}

Here, we study the statistical characteristic of the maximum of two modified generalized gamma random variates $\gamma_{\mathrm{M}}$ which is given by

$$
\gamma_{\mathrm{M}}=\max \left(\gamma_{a}, \gamma_{b}\right),
$$

assuming that $\gamma_{a}$ and $\gamma_{b}$ are independent not necessarily identically distributed (i.n.i.d) random variables (RVs).

1) Cumulative Distribution Function: The CDF can be easily derived as

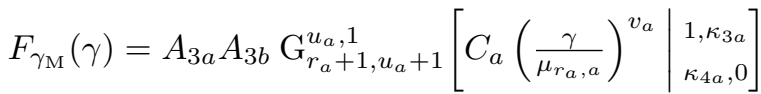

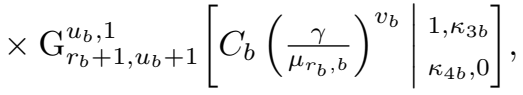

where $A_{3 i}=\frac{\xi_{i}^{2} \sigma_{i}^{\beta_{1 i}-\frac{1}{2}} \lambda_{i}^{\beta_{2 i}-\frac{1}{2}}(2 \pi)^{1-\frac{r_{i}\left(\lambda_{i}+\sigma_{i}\right)}{2}} r_{i}^{\beta_{1 i}+\beta_{2 i}-2}}{\alpha_{2 i} \lambda_{i} \Gamma\left(\beta_{1 i}\right) \Gamma\left(\beta_{2 i}\right)}$. For the case of Gamma-Gamma turbulence model, we have $A_{3 i}=$ $\xi_{i}^{2} r_{i}^{\alpha_{1}+\beta_{1}-2}$

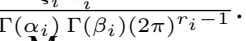

Moreover, an asymptotic expression can be obtained via the expansion of the Meijer's G-function [39, Eq. (26)] as $C_{i}\left(\frac{\gamma}{\mu_{r_{i}, i}}\right)^{v_{i}} \rightarrow 0$ (i.e high SNR) as

$$
\begin{aligned}
& \tilde{F}_{\gamma_{\mathrm{M}}}(\gamma) \underset{\mu_{r_{i}, i}>>1}{\approx} A_{3 a} A_{3 b} \sum_{k=1}^{u_{a}} \sum_{x=1}^{u_{b}}\left(\frac{\gamma^{v_{a}} C_{a}}{\mu_{r_{a}, a}^{v_{a}}}\right)^{\kappa_{4 a, k}}\left(\frac{\gamma^{v_{b}} C_{b}}{\mu_{r_{b}, b}^{v_{b}}}\right)^{\kappa_{4 b, x}} \\
& \times \frac{\prod_{l=1 ; l \neq k}^{u_{a}} \Gamma\left(\kappa_{4 a, l}-\kappa_{4 a, k}\right) \prod_{l=1 ; l \neq x}^{u_{b}} \Gamma\left(\kappa_{4 b, l}-\kappa_{4 b, x}\right)}{\kappa_{4 a, k} \kappa_{4 b, x} \prod_{l=1}^{r_{a}} \Gamma\left(\kappa_{3 a, l}-\kappa_{4 a, k}\right) \prod_{l=1}^{r_{b}} \Gamma\left(\kappa_{3 b, l}-\kappa_{4 b, k}\right)} .
\end{aligned}
$$


The asymptotic expression in (5) is simple and is in terms of elementary functions that can be evaluated using any computer software.

2) Probability Density Function: The PDF is then obtained by differentiating (4) with respect to $\gamma$ yielding

$$
\begin{aligned}
& f_{\gamma_{\mathrm{M}}}(\gamma)=\frac{A_{3 a} A_{3 b}}{\gamma}\left(v_{b} \mathrm{G}_{r_{b}, u_{b}}^{u_{b}, 0}\left[\begin{array}{l|l|l}
C_{b}\left(\frac{\gamma}{\mu_{r_{b}, b}}\right)^{v_{b}} & \kappa_{3 b} \\
\kappa_{4 b}
\end{array}\right]\right.
\end{aligned}
$$

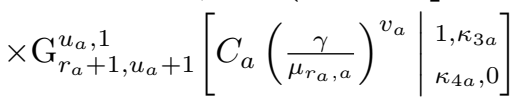

$$
\begin{aligned}
& +v_{a} \mathrm{G}_{r_{a}, u_{a}}^{u_{a}, 0}\left[C_{a}\left(\frac{\gamma}{\mu_{r_{a}, a}}\right)^{v_{a}} \mid \begin{array}{c|c}
\kappa_{3 a} \\
\kappa_{4 a}
\end{array}\right] \\
& \left.\times \mathrm{G}_{r_{b}+1, u_{b}+1}^{u_{b}, 1}\left[\begin{array}{l|l}
C_{b}\left(\frac{\gamma}{\mu_{r_{b}, b}}\right)^{v_{b}} & 1, \kappa_{3 b} \\
\kappa_{4 b}, 0
\end{array}\right]\right) .
\end{aligned}
$$

An asymptotic expression of the PDF can be obtained by the same way done earlier for the CDF. In other words, by using the expansion of the Meijer's G-function, we can reach to an asymptotic result as

$$
\begin{aligned}
& \tilde{f}_{\gamma_{\mathrm{M}}}(\gamma) \underset{\mu_{r_{i}, i}>>1}{\approx} A_{3 a} A_{3 b} \sum_{k=1}^{u_{a}} \sum_{x=1}^{u_{b}}\left(\frac{C_{a}}{\mu_{r_{a}, a}^{v_{a}}}\right)^{\kappa_{4 a, k}}\left(\frac{C_{b}}{\mu_{r_{b}, b}^{v_{b}}}\right)^{\kappa_{4 b, x}} \\
& \times \frac{\prod_{l=1 ; l \neq k}^{u_{a}} \Gamma\left(\kappa_{4 a, l}-\kappa_{4 a, k}\right) \prod_{l=1 ; l \neq x}^{u_{b}} \Gamma\left(\kappa_{4 b, l}-\kappa_{4 b, x}\right)}{\prod_{l=1}^{r_{b}} \Gamma\left(\kappa_{3 b, l}-\kappa_{4 b, x}\right) \prod_{l=1}^{r_{a}} \Gamma\left(\kappa_{3 a, l}-\kappa_{4 a, k}\right)} \\
& \times\left(\frac{v_{b}}{\kappa_{4 a, k}}+\frac{v_{a}}{\kappa_{4 b, x}}\right) \gamma^{v_{a} \kappa_{4 a, k}+v_{b} \kappa_{4 b, x}-1} .
\end{aligned}
$$

Derived PDFs were verified via Monte-Carlo simulations as

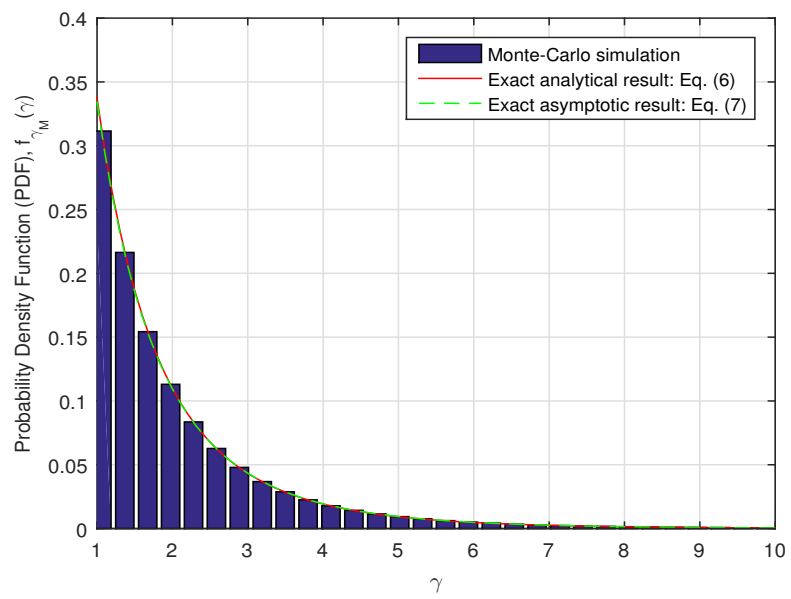

Figure 1: PDF validation for the maximum of two double GG variates

shown in Fig. 1.

3) Moment Generating Function: The MGF defined as $\mathcal{M}_{\gamma_{\mathrm{M}}}(s) \triangleq \mathbb{E}\left[e^{-\gamma_{\mathrm{M}} s}\right]$ can be expressed in terms of CDF using integration by parts as [40]

$$
\mathcal{M}_{\gamma_{\mathrm{M}}}(s)=s \int_{0}^{\infty} \exp (-\gamma s) F_{\gamma_{\mathrm{M}}}(\gamma) d \gamma
$$

Using the property $\frac{1}{C} \mathrm{G}_{p, q}^{m, n}\left[z^{1 / C} \mid \begin{array}{l}\mathbf{a} \\ \mathbf{b}\end{array}\right]=\mathrm{H}_{p, q}^{m, n}\left[z \mid \begin{array}{c}(a, C) \\ (b, C)\end{array}\right]$, we transform each term to the Fox's H-function that can be evaluated using Mathematica [41]. As such, the CDF in (4) can be written as

$$
\begin{aligned}
F_{\gamma_{\mathrm{M}}}(\gamma) & =\frac{A_{3 a} A_{3 b}}{v_{a} v_{b}} \mathrm{H}_{r_{a}+1, u_{a}+1}^{u_{a}, 1}\left[\frac{C_{a}^{1 / v a} \gamma}{\mu_{r_{a}, a}} \mid \begin{array}{l}
\left(\kappa_{5 a}, 1 / v a\right) \\
\left(\kappa_{6 a}, 1 / v a\right)
\end{array}\right] \\
& \times \mathrm{H}_{r_{b}+1, u_{b}+1}^{u_{b}, 1}\left[\frac{C_{b}^{1 / v b} \gamma}{\mu_{r_{b}, b}} \mid \begin{array}{l}
\left(\kappa_{5 b}, 1 / v b\right) \\
\left(\kappa_{6 b}, 1 / v b\right)
\end{array}\right],
\end{aligned}
$$

where $\kappa_{5 i}=1, \kappa_{3 i}$ and $\kappa_{6 i}=\kappa_{4 i}, 0$. Afterward, we apply the identity [42, Eq. (2.2)], after inserting (9) in (8) to obtain

$$
\begin{aligned}
& \mathcal{M}_{\gamma_{\mathrm{M}}}(s)=\frac{A_{3 a} A_{3 b}}{v_{a} v_{b}}
\end{aligned}
$$

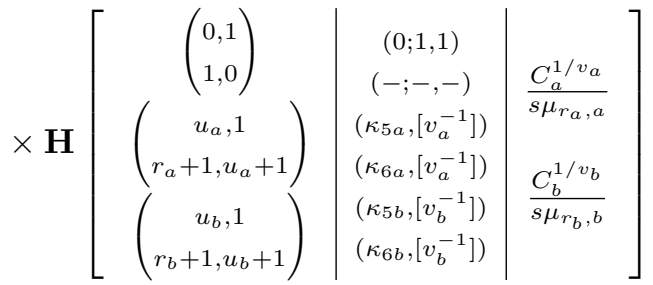

where $\mathbf{H}[$.$] is the bivariate \mathrm{H}$-function defined in [42]. It can be evaluated efficiently using MATHEMATICA ${ }^{\circledR}$ implementation in [43] or MATLAB ${ }^{\circledR}$ implementation in [44]. In this work, we have implemented the bivariate $\mathrm{H}$-function in MATHEMATICA ${ }^{\circledR}$ and easily evaluated the expressions.

Simplified formula can be obtained if solving (8) but using the asymptotic CDF (5) to reach to the following result

$$
\begin{aligned}
& \tilde{\mathcal{M}}_{\gamma_{\mathrm{M}}}(s) \underset{\mu_{r_{i}, i}>>1}{\approx} A_{3 a} A_{3 b} \sum_{k=1}^{u_{a}} \sum_{x=1}^{u_{b}}\left(\frac{C_{a}}{\mu_{r_{a}, a}^{v_{a}}}\right)^{\kappa_{4 a, k}}\left(\frac{C_{b}}{\mu_{r_{b}, b}^{v_{b}}}\right)^{\kappa_{4 b, x}} \\
& \times \frac{\prod_{l=1 ; l \neq k}^{u_{a}} \Gamma\left(\kappa_{4 a, l}-\kappa_{4 a, k}\right) \prod_{l=1 ; l \neq x}^{u_{b}} \Gamma\left(\kappa_{4 b, l}-\kappa_{4 b, x}\right)}{\prod_{l=1}^{r_{a}} \Gamma\left(\kappa_{3 a, l}-\kappa_{4 a, k}\right) \prod_{l=1}^{r_{b}} \Gamma\left(\kappa_{3 b, l}-\kappa_{4 b, k}\right)} \\
& \times \frac{\Gamma\left(v_{a} \kappa_{4 a, k}+v_{b} \kappa_{4 b, x}+1\right) s^{-\left(v_{a} \kappa_{4 a, k}+v_{b} \kappa_{4 b, x}\right)}}{\kappa_{4 a, k} \kappa_{4 b, x}} .
\end{aligned}
$$

Setting the same special parameters listed in Sec. II for the Gamma-Gamma case, the MGF can be expressed as

$$
\begin{aligned}
& \mathcal{M}_{\gamma_{\mathrm{M}}}^{*}(s)=A_{3 a} A_{3 b} \\
& \times \mathrm{G}_{1,0: 3 r_{a}+1,3 r_{a}+1: r_{b}+1,3 r_{b}+1}^{1,0: 1}\left[\begin{array}{c|c|c|c}
1 & \kappa_{5 a} & \kappa_{5 b} & \frac{C_{a}}{s r_{r_{a}, a}}, \frac{C_{b}}{s \mu_{r_{b}, b}} \\
- & \kappa_{6 a} & \kappa_{6 b}
\end{array},\right.
\end{aligned}
$$

in agreement with $[4, \mathrm{Eq} .(8)]$ where $\mathrm{G}_{-,-,-,-,:-,-,-}^{-,-}[$.$] is the ex-$ tended generalized bivariate Meijer's G-function (EGBMGF) that can be evaluated using the code given in [43, Table II].

4) Moments: The moments is defined as

$$
\mathbb{E}\left[\gamma^{n}\right] \triangleq \int_{0}^{\infty} \gamma^{n} f_{\gamma}(\gamma) d \gamma
$$


Placing (6) in (13) and utilizing equation [45, Eq. (1.7)], we obtain the following

$$
\begin{aligned}
& \mathbb{E}\left[\gamma_{\mathrm{M}}^{n}\right]=A_{3 a} A_{3 b}\left[\left(\frac{\mu_{r_{a}, a}}{C_{a}^{1 / v_{a}}}\right)^{n} v_{b}\right. \\
& \times \mathrm{H}_{u_{a}+r_{b}+1, u_{b}+r_{a}+1}^{u_{b}+1, u_{a}}\left[\left(\frac{C_{b}^{1 / v_{b}} \mu_{r_{a}, a}}{C_{a}^{1 / v_{a}} \mu_{r_{b}, b}}\right)^{v_{a} v_{b}} \mid \begin{array}{l}
\left(\kappa_{7 a}, v_{b}\right),\left(\kappa_{3 b}, v_{a}\right) \\
\left(\kappa_{4 b}, v_{a}\right),\left(\kappa_{8 a}, v_{b}\right)
\end{array}\right] \\
& +\left(\frac{\mu_{r_{b}, b}}{C_{b}^{1 / v_{b}}}\right)^{n} v_{a} \\
& \left.\times \mathrm{H}_{u_{b}+r_{a}+1, u_{a}+r_{b}+1}^{u_{a}+1, u_{b}}\left[\left(\frac{C_{a}^{1 / v_{a}} \mu_{r_{b}, b}}{C_{b}^{1 / v_{b} \mu_{r_{a}, a}}}\right)^{v_{a} v_{b}} \mid \begin{array}{l}
\left(\kappa_{7 b}, v_{a}\right),\left(\kappa_{3 a}, v_{b}\right) \\
\left(\kappa_{4 a}, v_{b}\right),\left(\kappa_{8 b}, v_{a}\right)
\end{array}\right]\right] .
\end{aligned}
$$

where $\kappa_{7 i}=1-\kappa_{6 i}-\frac{n}{v_{i}}$ and $\kappa_{8 i}=1-\kappa_{5 i}-\frac{n}{v_{i}}$. Setting the same parameters listed in Sec. II, (14) simplifies to

$$
\begin{aligned}
& \mathbb{E}\left[\gamma_{\mathrm{M}}^{n}\right]^{*}=A_{3 a} A_{3 b} \\
& \times\left[\left(\frac{\mu_{r_{a}, a}}{C_{a}}\right)^{n} \mathrm{G}_{3 r_{a}+r_{b}+1,3 r_{b}+r_{a}+1}^{3 r_{b}+1,3 r_{a}}\left[\begin{array}{l|l}
C_{b} \mu_{r_{a}, a} & \kappa_{7 a}, \kappa_{3 b} \\
C_{a} \mu_{r_{b}, b} & \kappa_{4 b}, \kappa_{8 a}
\end{array}\right]\right. \\
& \left.+\left(\frac{\mu_{r_{b}, b}}{C_{b}}\right)^{n} \mathrm{G}_{3 r_{b}+r_{a}+1,3 r_{a}+r_{b}+1}^{3 r_{a}+1,3 r_{b}}\left[\begin{array}{cc|c}
\frac{C_{a} \mu_{r_{b}, b}}{C_{b} \mu_{r_{a}, a}} & \kappa_{7 b}, \kappa_{3 a} \\
\kappa_{4 a}, \kappa_{8 b}
\end{array}\right]\right],
\end{aligned}
$$

in agreement with [4, Eq. (10)].

\section{B. Minimum of Two Variates}

In this section, we list the statistical properties of the minimum of two modified generalized gamma random variables $\gamma_{\mathrm{m}}$ given by

$$
\gamma_{\mathrm{m}}=\min \left(\gamma_{1}, \gamma_{2}\right)
$$

1) Cumulative Distribution Function: The CDF is given by

$$
\begin{aligned}
F_{\gamma_{\mathrm{m}}}(\gamma) & =1-\operatorname{Pr}\left(\min \left(\gamma_{1}, \gamma_{2}\right)>\gamma\right) \\
& =F_{\gamma_{1}}(\gamma)+F_{\gamma_{2}}(\gamma)-F_{\gamma_{1}}(\gamma) F_{\gamma_{2}}(\gamma),
\end{aligned}
$$

under the assumption that $\gamma_{1}$ and $\gamma_{2}$ are i.n.i.d. Substituting (2) in (17), we obtain

$$
\begin{aligned}
F_{\gamma_{\mathrm{m}}}(\gamma) & =A_{3 a} \mathrm{G}_{r_{a}+1, u_{a}+1}^{u_{a}, 1}\left[C_{a}\left(\frac{\gamma}{\mu_{r_{a}, a}}\right)^{v_{a}} \mid \begin{array}{c}
1, \kappa_{3 a} \\
\kappa_{4 a}, 0
\end{array}\right] \\
& +A_{3 b} \mathrm{G}_{r_{b}+1, u_{b}+1}^{u_{b}, 1}\left[C_{b}\left(\frac{\gamma}{\mu_{r_{b}, b}}\right)^{v_{b}} \mid \begin{array}{c}
1, \kappa_{3 b} \\
\kappa_{4 b}, 0
\end{array}\right] \\
& -A_{3 a} A_{3 b} \mathrm{G}_{r_{a}+1, u_{a}+1}^{u_{a}, 1}\left[C_{a}\left(\frac{\gamma}{\mu_{r_{a}, a}}\right)^{v_{a}} \mid \begin{array}{c}
1, \kappa_{3 a} \\
\kappa_{4 a}, 0
\end{array}\right] \\
& \times \mathrm{G}_{r_{b}+1, u_{b}+1}^{u_{b}, 1}\left[C_{b}\left(\frac{\gamma}{\mu_{r_{b}, b}}\right)^{v_{b}} \mid \begin{array}{c}
1, \kappa_{3 b} \\
\kappa_{4 b}, 0
\end{array}\right] .
\end{aligned}
$$

In addition, an asymptotic expression can be obtained via the expansion of the Meijer's G function as

$$
\begin{aligned}
\tilde{F}_{\gamma_{\mathrm{m}}}(\gamma) \underset{\mu_{r_{i}, i}>>1}{\approx} A_{3 a} \sum_{k=1}^{u_{a}}\left(\frac{\gamma^{v_{a}} C_{a}}{\mu_{r_{a}, a}^{v_{a}}}\right)^{\kappa_{4 a, k}} \\
\quad \times \frac{\prod_{l=1 ; l \neq k}^{u_{a}} \Gamma\left(\kappa_{4 a, l}-\kappa_{4 a, k}\right)}{\kappa_{4 a, k} \prod_{l=1}^{r_{a}} \Gamma\left(\kappa_{3 a, l}-\kappa_{4 a, k}\right)}+A_{3 b} \sum_{x=1}^{u_{b}}\left(\frac{\gamma^{v_{b}} C_{b}}{\mu_{r_{b}, b}^{v_{b}}}\right)^{\kappa_{4 b, x}} \\
\quad \times \frac{\prod_{l=1 ; l \neq x}^{u_{b}} \Gamma\left(\kappa_{4 b, l}-\kappa_{4 b, x}\right)}{\kappa_{4 b, x} \prod_{l=1}^{r_{b}} \Gamma\left(\kappa_{3 b, l}-\kappa_{4 b, x}\right)}-\tilde{F}_{\gamma_{\mathrm{M}}}(\gamma) .
\end{aligned}
$$

2) Probability Density Function: Differentiating (18) with respect to $\gamma$, a closed-form expression of the PDF of $\gamma_{\mathrm{m}}$ is obtained as

$$
\begin{aligned}
& f_{\gamma_{\mathrm{m}}}(\gamma)=\frac{A_{3 a}}{\gamma} v_{a} \mathrm{G}_{r_{a}, u_{a}}^{u_{a}, 0}\left[C_{a}\left(\frac{\gamma}{\mu_{r_{a}, a}}\right)^{v_{a}} \mid \begin{array}{c}
\kappa_{3 a} \\
\kappa_{4 a}
\end{array}\right] \\
& +\frac{A_{3 b}}{\gamma} v_{b} \mathrm{G}_{r_{b}, u_{b}}^{u_{b}, 0}\left[C_{b}\left(\frac{\gamma}{\mu_{r_{b}, b}}\right)^{v_{b}} \mid \begin{array}{c}
\kappa_{3 b} \\
\kappa_{4 b}
\end{array}\right]-f_{\gamma_{M}}(\gamma)
\end{aligned}
$$

where $f_{\gamma_{M}}(\gamma)$ is defined in (6). Similarly, an asymptotic expression of the PDF can be obtained when expanding the Meijer's G-function leading to

$$
\begin{aligned}
& \tilde{f}_{\gamma_{\mathrm{m}}}(\gamma) \underset{\mu_{r_{i}, i}>>1}{\approx} A_{3 a} v_{a} \sum_{k=1}^{u_{a}}\left(\frac{C_{a}}{\mu_{r_{a}, a}^{v_{a}}}\right)^{\kappa_{4 a, k}} \gamma^{v_{a} \kappa_{4 a, k}-1} \\
& \times \frac{\prod_{l=1 ; l \neq k}^{u_{a}} \Gamma\left(\kappa_{4 a, l}-\kappa_{4 a, k}\right)}{\prod_{l=1}^{r_{a}} \Gamma\left(\kappa_{3 a, l}-\kappa_{4 a, k}\right)} \\
& +A_{3 b} v_{b} \sum_{x=1}^{u_{b}}\left(\frac{C_{b}}{\mu_{r_{b}, b}^{v_{b}}}\right)^{\kappa_{4 b, x}} \gamma^{v_{b} \kappa_{4 b, x}-1} \\
& \times \frac{\prod_{l=1 ; l \neq x}^{u_{b}} \Gamma\left(\kappa_{4 b, l}-\kappa_{4 b, x}\right)}{\prod_{l=1}^{r_{b}} \Gamma\left(\kappa_{3 b, l}-\kappa_{4 b, x}\right)}-\tilde{f}_{\gamma_{\mathrm{M}}}(\gamma) .
\end{aligned}
$$

Monte-Carlo simulations were utilized again to verify the

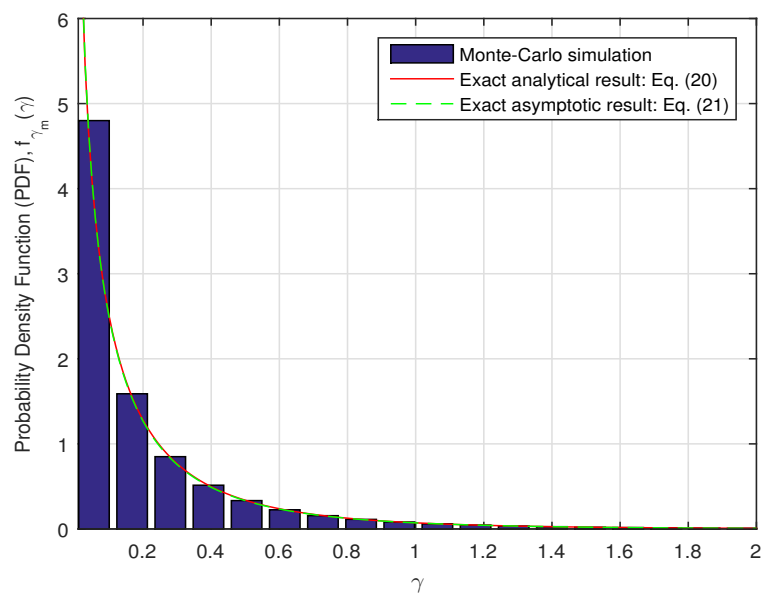

Figure 2: PDF validation for the minimum of two double GG variates

PDFs as in Fig. 2.

3) Moment Generating Function: Placing (18) in the definition of the MGF, and utilizing Eq. (10) and [46, Eq. (07.34.21.0088.01)], the MGF can be expressed as

$$
\begin{aligned}
& \mathcal{M}_{\gamma_{\mathrm{m}}}(s)=\frac{A_{3 a} \sqrt{v_{a}}}{(2 \pi)^{\frac{v_{a}-1}{2}}} \mathrm{G}_{r_{a}+v_{a}, u_{a}}^{u_{a}, v_{a}}\left[C_{a}\left(\frac{v_{a}}{s \mu_{r_{a}, a}}\right)^{v_{a}} \mid \begin{array}{c}
\Delta\left(v_{a}, 1\right), \kappa_{3 a} \\
\kappa_{4 a}
\end{array}\right] \\
& +\frac{A_{3 b \sqrt{v_{b}}}}{(2 \pi)^{\frac{v_{b}-1}{2}}} \mathrm{G}_{r_{b}+v_{b}, u_{b}}^{u_{b}, v_{b}}\left[C_{b}\left(\frac{v_{b}}{s \mu_{r_{b}, b}}\right)^{v_{b}} \mid \begin{array}{c}
\Delta\left(v_{b}, 1\right), \kappa_{3 b} \\
\kappa_{4 b}
\end{array}\right]-\mathcal{M}_{\gamma_{\mathrm{M}}}(s) .
\end{aligned}
$$

More simplified expression for the MGF can be obtained if the expansion of the Meijer's G-function were utilized in a 
similar way to find the MGF of the maximum

$$
\begin{aligned}
& \tilde{\mathcal{M}}_{\gamma_{\mathrm{m}}}(s) \underset{\mu_{r_{i}, i}>>1}{\approx} A_{3 a} v_{a} \sum_{k=1}^{u_{a}}\left(\frac{C_{a}}{\left(s \mu_{r_{a}, a}\right)^{v_{a}}}\right)^{\kappa_{4 a, k}} \Gamma\left(v_{a} \kappa_{4 a, k}\right) \\
& \times \frac{\prod_{l=1 ; l \neq k}^{u_{a}} \Gamma\left(\kappa_{4 a, l}-\kappa_{4 a, k}\right)}{\prod_{l=1}^{r_{a}} \Gamma\left(\kappa_{3 a, l}-\kappa_{4 a, k}\right)}+A_{3 b} v_{b} \sum_{x=1}^{u_{b}}\left(\frac{C_{b}}{\left(s \mu_{r_{b}, b}\right)^{v_{b}}}\right)^{\kappa_{4 b, x}} \\
& \times \frac{\Gamma\left(v_{b} \kappa_{4 b, x}\right) \prod_{l=1 ; l \neq x}^{u_{b}} \Gamma\left(\kappa_{4 b, l}-\kappa_{4 b, x}\right)}{\prod_{l=1}^{r_{b}} \Gamma\left(\kappa_{3 b, l}-\kappa_{4 b, x}\right)}-\tilde{\mathcal{M}}_{\gamma_{\mathrm{M}}}(s) .
\end{aligned}
$$

The MGF for Gamma-Gamma model can be obtained by setting the same parameters listed in Sec. II

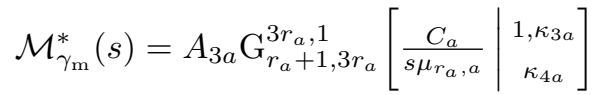

$$
\begin{aligned}
& +A_{3 b} \mathrm{G}_{r_{b}+1,3 r_{b}}^{3 r_{b}, 1}\left[\begin{array}{c|c}
\frac{C_{b}}{s \mu_{r_{b}, b}} & 1, \kappa_{3 b} \\
\kappa_{4 b}
\end{array}\right]-A_{3 a} A_{3 b} \\
& \times \mathrm{G}_{1,0: r_{a}+1,3 r_{a}+1: r_{b}+1,3 r_{b}+1}^{1,0: 3 r_{a}, 1: 3 r_{b}, 1}\left[\begin{array}{c|c|c|c}
1 & \kappa_{5 a} & \kappa_{5 b} & \frac{C_{a}}{s \mu_{r_{a}, a}}, \frac{C_{b}}{s \mu_{r_{b}, b}}
\end{array}\right] .
\end{aligned}
$$

in agreement with [4, Eq. (15)].

4) Moments: Substituting (20) in the definition of the moments (13) and utilizing [46, Eq. (2.25.2.1)] and Eq. (14), we obtain the moments as

$$
\begin{aligned}
& \mathbb{E}\left[\gamma_{\mathrm{m}}^{n}\right]=A_{3 a}\left(\frac{\mu_{r_{a}, a}}{C_{a}^{1 / v_{a}}}\right)^{n} \Gamma\left(\begin{array}{c}
\kappa_{4 a}+\frac{n}{v_{a}} \\
\kappa_{3 a}+\frac{n}{v_{a}}
\end{array}\right)+A_{3 b}\left(\frac{\mu_{r_{b}, b}}{C_{b}^{1 / v_{b}}}\right)^{n} \\
& \times \Gamma\left(\begin{array}{l}
\kappa_{4 b}+\frac{n}{v_{b}} \\
\kappa_{3 b}+\frac{n}{v_{b}}
\end{array}\right)-\mathbb{E}\left[\begin{array}{c}
\left.\gamma_{\mathrm{M}}^{n}\right]
\end{array}\right.
\end{aligned}
$$

where $\Gamma\left(\begin{array}{l}\mathbf{x} \\ \mathbf{y}\end{array}\right)=\prod_{i=1}^{m} \Gamma\left(x_{i}\right) / \prod_{i=1}^{n} \Gamma\left(y_{i}\right)$ such that $m$ and $n$ are the lengths of $x$ and $y$, respectively.

In the Gamma-Gamma case, (25) simplifies to

$$
\begin{aligned}
& \mathbb{E}\left[\gamma_{m}^{n}\right]^{*}=A_{3 a}\left(\frac{\mu_{r_{a}, a}}{C_{a}^{1 / v_{a}}}\right)^{n} \Gamma\left(\begin{array}{c}
\kappa_{4 a}+n \\
\kappa_{3 a}+n
\end{array}\right) \\
& +A_{3 b}\left(\frac{\mu_{r_{b}, b}}{C_{b}^{1 / v_{b}}}\right)^{n} \Gamma\left(\begin{array}{l}
\kappa_{4 b}+n \\
\kappa_{3 b}+n
\end{array}\right) \\
& \text { - } A_{3 a} A_{3 b}\left[\left(\frac{\mu_{r_{a}, a}}{C_{a}}\right)^{n} \mathrm{G}_{3 r_{a}+r_{b}+1,3 r_{b}+r_{a}+1}^{3 r_{b}+1,3 r_{a}}\left[\begin{array}{l|l}
\frac{C_{b} \mu_{r_{a}, a}}{C_{a} \mu_{r_{b}, b}} & \kappa_{7 a}, \kappa_{3 b} \\
\kappa_{4 b}, \kappa_{8 a}
\end{array}\right]\right. \\
& \left.+\left(\frac{\mu_{r_{b}, b}}{C_{b}}\right)^{n} \mathrm{G}_{3 r_{b}+r_{a}+1,3 r_{a}+r_{b}+1}^{3 r_{a}+1, r_{b}}\left[\begin{array}{l|l}
C_{a} \mu_{r_{b}, b} & \kappa_{7 b}, \kappa_{3 a} \\
\hline C_{b} \mu_{r_{a}, a} & \kappa_{4 a}, \kappa_{8 b}
\end{array}\right]\right] .
\end{aligned}
$$

as found in [4, Eq. (16)].

\section{ApPliCATiOns}

\section{A. Performance of Dual-Branch SC Systems}

Based on our results in Section III-A, we now study the performance of the selection combining scheme over dualbranch FSO links.

1) Outage Probability: The outage probability $P_{\text {out }}$ is defined as follows:

$$
P_{\text {out }}=\operatorname{Pr}\left[\gamma<\gamma_{t h}\right]=F_{\gamma}\left(\gamma_{t h}\right) .
$$

Accordingly, the outage probability for the maximum SNR of two links is obtained by substituting (4) into (27).
2) Average Bit Error Rate: Using the definition of the average BER [43, Eq. (12)]

$$
\bar{P}=\frac{q^{p}}{2 \Gamma(p)} \int_{0}^{\infty} \exp (-q \gamma) \gamma^{p-1} F_{\gamma}(\gamma) d \gamma
$$

and placing (4) into (28) then utilizing the identity [42, Eq. (2.2)], we obtain $\bar{P}_{\mathrm{SC}}$ as

$$
\begin{aligned}
\bar{P}_{\mathrm{SC}} & =\frac{A_{3 a} A_{3 b}}{2 \Gamma(p) v_{a} v_{b}} \\
& \times \mathbf{H}\left[\begin{array}{c|c|c}
\left(\begin{array}{c}
0,1 \\
1,0
\end{array}\right) & \begin{array}{c}
(1-p ; 1,1) \\
(-;-,-)
\end{array} & \frac{C_{a}^{1 / v_{a}}}{q \mu_{r_{a}, a}} \\
\left(\begin{array}{c}
u_{a}, 1 \\
r_{a}+1, u_{a}+1
\end{array}\right) & \begin{array}{c}
\left(\kappa_{5 a},\left[v_{a}^{-1}\right]\right) \\
\left(\kappa_{6 a},\left[v_{a}^{-1}\right]\right)
\end{array} & C_{b}^{1 / v_{b}} \\
\left(\begin{array}{c}
u_{b}, 1 \\
r_{b}+1, u_{b}+1
\end{array}\right) & \left(\kappa_{b b},\left[v_{b}^{-1}\right]\right) & \frac{q \mu_{r_{b}, b}}{\left(\kappa^{-1}\right]}
\end{array}\right],
\end{aligned}
$$

where $p$ and $q$ indicate different modulation schemes parameters [40, Table I].

An asymptotic expression of $\bar{P}$ at high SNR can be derived as

$$
\bar{P} \underset{\mu_{r_{i}, i}>>1}{\approx} \frac{q^{p}}{2 \Gamma(p)} \int_{0}^{\infty} \exp (-q \gamma) \gamma^{p-1} \tilde{F}(\gamma) d \gamma
$$

Solving the integration leads to the following:

$$
\begin{aligned}
& \bar{P}_{\mathrm{SC}} \underset{\mu_{r_{i}, i}>>1}{\approx} \frac{A_{3 a} A_{3 b}}{2 \Gamma(p)} \sum_{k=1}^{u_{a}} \sum_{x=1}^{u_{b}}\left(\frac{C_{a}}{\mu_{r_{a}, a}^{v_{a}}}\right)^{\kappa_{4 a, k}}\left(\frac{C_{b}}{\mu_{r_{b}, b}^{v_{b}}}\right)^{\kappa_{4 b, x}} \\
& \times \frac{\prod_{l=1 ; l \neq k}^{u_{a}} \Gamma\left(\kappa_{4 a, l}-\kappa_{4 a, k}\right) \prod_{l=1 ; l \neq x}^{u_{b}} \Gamma\left(\kappa_{4 b, l}-\kappa_{4 b, x}\right)}{\prod_{l=1}^{r_{a}} \Gamma\left(\kappa_{3 a, l}-\kappa_{4 a, k}\right) \prod_{l=1}^{r_{b}} \Gamma\left(\kappa_{3 b, l}-\kappa_{4 b, k}\right)} \\
& \times \frac{\Gamma\left(v_{a} \kappa_{4 a, k}+v_{b} \kappa_{4 b, x}+p\right) q^{-\left(v_{a} \kappa_{4 a, k}+v_{b} \kappa_{4 b, x}\right)}}{\kappa_{4 a, k} \kappa_{4 b, x}} .
\end{aligned}
$$

Diversity and coding gain can be extracted from (31) if the expression consists of only one term. In particular when the BER is the form $\bar{P} \approx\left(G_{c} \mu\right)^{-G_{d}}$ [47] where $G_{c}$ and $G_{d}$ are the diversity and coding gain respectively, and $\mu$ is the average SNR. Upon our observation in other study [37], this is possible if the absolute difference between $\left\{\frac{\xi_{i}^{2}}{v_{i} r_{i}}, \frac{\beta_{1 i}}{r_{i} \sigma_{i}}, \frac{\beta_{2 i}}{r_{i} \lambda_{i}}\right\} \in \kappa_{4 i}$ is significance. If so, then the summation in (31) reduces to a single dominant term results from the $\min \left(\frac{\xi_{i}^{2}}{v_{i} r_{i}}, \frac{\beta_{1 i}}{r_{i} \sigma_{i}}, \frac{\beta_{2 i}}{r_{i} \lambda_{i}}\right)$. In this case, the diversity and coding gain can be written as

$G_{d}=v_{a} \kappa_{4 a, k}+v_{b} \kappa_{4 b, x}$,

$G_{c}=q\left(\frac{A_{3 a} A_{3 b} C_{a}{ }^{\kappa_{4 a, k}} C_{b}{ }^{\kappa_{4 b, x}} \Gamma\left(v_{a} \kappa_{4 a, k}+v_{b} \kappa_{4 b, x}+p\right)}{2 \Gamma(p) \kappa_{4 a, k} \kappa_{4 b, x}}\right.$

$\left.\times \frac{\prod_{l=1 ; l \neq k}^{u_{a}} \Gamma\left(\kappa_{4 a, l}-\kappa_{4 a, k}\right) \prod_{l=1 ; l \neq x}^{u_{b}} \Gamma\left(\kappa_{4 b, l}-\kappa_{4 b, x}\right)}{\prod_{l=1}^{r_{a}} \Gamma\left(\kappa_{3 a, l}-\kappa_{4 a, k}\right) \prod_{l=1}^{r_{b}} \Gamma\left(\kappa_{3 b, l}-\kappa_{4 b, k}\right)}\right)^{-1 / G_{d}}$,

where $k \in\left\{1, r_{a}+1, r_{a}+r_{a} \sigma_{a}+1\right\}$ and $x \in\left\{1, r_{b}+1, r_{b}+\right.$ $\left.r_{b} \sigma_{b}+1\right\}$. 
For the Gamma-Gamma case, we have

$$
\begin{aligned}
& \bar{P}_{\mathrm{SC}}^{*}=\frac{A_{3 a} A_{3 b}}{2 \Gamma(p)} \\
& \times \mathrm{G}_{1,0: r_{a}+1,3 r_{a}+1: r_{b}+1,3 r_{b}+1}^{1,0: 3 r_{a}, 1: 3 r_{b}, 1}\left[\begin{array}{c|c|c|c}
p & \kappa_{5 a} & \kappa_{5 b} & \frac{C_{a}}{q \mu_{r_{a}, a}}, \frac{C_{b}}{q \mu_{r_{b}, b}}
\end{array}\right],
\end{aligned}
$$

in agreement with [4, Eq. (19)]

3) Ergodic Capacity: The ergodic capacity in FSO systems is defined as

$$
\bar{C} \triangleq \mathbb{E}\left[\log _{2}(1+c \gamma)\right]=\int_{0}^{\infty} \log _{2}(1+c \gamma) f_{\gamma}(\gamma) d \gamma,
$$

where $c=1$ for heterodyne detection $r=1$ and $c=e / 2 \pi$ for IM/DD $r=2$. It is very important to note that the expression in (34) is exact for $r=1$ while it is a lower bound for the case of $r=2$ [48, Eq. (26)] [49, Eq. (7.43)]. $\log _{2}(1+c \gamma)$ is expressed as $\frac{\ln (1+\gamma)}{\ln (2)} \cdot \bar{C}_{\mathrm{SC}}$ is obtained by using (34) along with the identities $\ln (1+x)=\mathrm{G}_{2,2}^{1,2}\left[x \mid \begin{array}{l}1,1 \\ 1,0\end{array}\right]=\mathrm{H}_{2,2}^{1,2}\left[x \mid \begin{array}{l}(1,1),(1,1) \\ (1,1),(0,1)\end{array}\right]$ and [42, Eq. (2.3)] yielding

$$
\begin{aligned}
& \bar{C}_{\mathrm{SC}}=\frac{A_{3 a} A_{3 b}}{v_{a} v_{b} \ln (2)}
\end{aligned}
$$

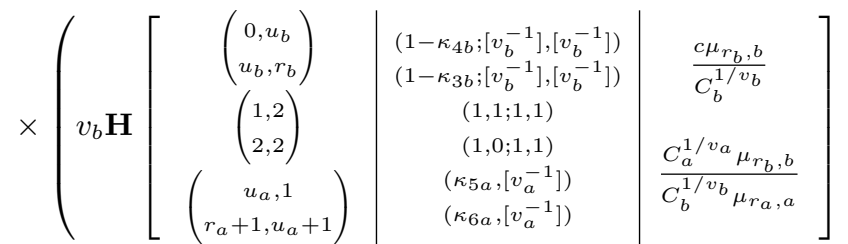

$$
\begin{aligned}
& \left.+v_{a} \mathbf{H}\left[\begin{array}{c|c|c}
\left(\begin{array}{c}
0, u_{a} \\
u_{a}, r_{a}
\end{array}\right) & \left(1-\kappa_{4 a} ;\left[v_{a}^{-1}\right],\left[v_{a}^{-1}\right]\right) & \frac{c \mu_{r_{a}, a}}{C_{a}^{1 / v_{a}}} \\
\left(\begin{array}{c}
1,2 \\
2,2
\end{array}\right) & \left(1-\kappa_{3 a} ;\left[v_{a}^{-1}\right],\left[v_{a}^{-1}\right]\right) & \\
(1,0 ; 1,1) & \frac{C_{b}^{1 / v_{b}} \mu_{r_{a}, a}}{C_{a}^{1 / v_{a}} \mu_{r_{b}, b}}
\end{array}\right]\right)
\end{aligned}
$$

For the Gamma-Gamma case, we have

$$
\begin{aligned}
& \bar{C}_{\mathrm{SC}}^{*}=\frac{A_{3 a} A_{3 b}}{\ln (2)}
\end{aligned}
$$

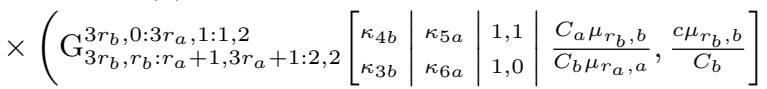

$$
\begin{aligned}
& \left.+\mathrm{G}_{3 r_{a}, r_{a}: r_{b}+1,3 r_{b}+1: 2,2}^{3 r_{a}, 0: 3 r_{b}, 1: 1,2}\left[\begin{array}{c|c|c|c}
\kappa_{4 a} & \kappa_{5 b} & 1,1 & \frac{B_{b} \mu_{r_{a}, a}}{B_{a} \mu_{r_{b}, b}}, \frac{c \mu_{r_{a}, a}}{\kappa_{a}}
\end{array}\right]\right) .
\end{aligned}
$$

in agreement with [4, Eq. (21)].

\section{B. Performance of Dual-Hop Relay Systems}

In this section, we present the performance analysis of a dual-hop (DH) FSO variable gain relay transmission systems whose end-to-end SNR is known to be given by $\gamma_{\mathrm{DH}}=$ $\left(\gamma_{1} \gamma_{2}\right) /\left(1+\gamma_{1}+\gamma_{2}\right)$ and which is typically approximated by $\gamma_{\mathrm{DH}} \simeq \min \left(\gamma_{1}, \gamma_{2}\right)$ [32]. Thus, results obtained in Section III-B facilitate the following analysis.

1) Outage Probability: The outage probability for the minimum SNR of two links is obtained by substituting (18) into (27).
2) Average Bit Error Rate: $\bar{P}_{\mathrm{DH}}$ is obtained by placing (18) into (28), and utilizing (29) and [43, 2.24.3.2] yielding

$$
\begin{aligned}
& \bar{P}_{\mathrm{DH}}=\frac{A_{3 a} v_{a}^{p-1 / 2}}{2 \Gamma(p)(2 \pi)^{\frac{v_{a}-1}{2}}} \\
& \times \mathrm{G}_{r_{a}+v_{a}+1, u_{a}+1}^{u_{a}, v_{a}+1}\left[\begin{array}{l|l}
C_{a}\left(\frac{v_{a}}{q \mu_{r_{a}, a}}\right)^{v_{a}} & \Delta\left(v_{a}, 1-p\right), \kappa_{5 a} \\
\kappa_{6 a}
\end{array}\right] \\
& +\frac{A_{3 b} v_{b}^{p-1 / 2}}{2 \Gamma(p)(2 \pi)^{\frac{v_{b}-1}{2}}} \mathrm{G}_{r_{b}+v_{b}+1, u_{b}+1}^{u_{b}, v_{b}+1}\left[\begin{array}{l|l}
C_{b}\left(\frac{v_{b}}{q \mu_{r_{b}, b}}\right)^{v_{b}} & \left.\begin{array}{c}
\Delta\left(v_{b}, 1-p\right), \kappa_{5 b} \\
\kappa_{6 b}
\end{array}\right]
\end{array}\right. \\
& -\bar{P}_{\mathrm{SC}} \text {. }
\end{aligned}
$$

Similar to the SC, we can obtain an asymptotic expression of the BER via Meijer's G-function expansion by solving (30) as follows:

$$
\begin{aligned}
& \bar{P}_{D H} \underset{\mu_{r_{i}, i}>>1}{\approx} \frac{A_{3 a}}{2 \Gamma(p)} \sum_{k=1}^{u_{a}}\left(\frac{C_{a}}{\left(q \mu_{r_{a}, a}\right)^{v_{a}}}\right)^{\kappa_{4 a, k}} \\
& \times \frac{\Gamma\left(v_{a} \kappa_{4 a, k}+p\right) \prod_{l=1 ; l \neq k}^{u_{a}} \Gamma\left(\kappa_{4 a, l}-\kappa_{4 a, k}\right)}{\kappa_{4 a, k} \prod_{l=1}^{r_{a}} \Gamma\left(\kappa_{3 a, l}-\kappa_{4 a, k}\right)} \\
& +\frac{A_{3 b}}{2 \Gamma(p)} \sum_{x=1}^{u_{b}}\left(\frac{C_{b}}{q \mu_{r_{b}, b} v_{b}}\right)^{\kappa_{4 b, x}} \\
& \times \frac{\Gamma\left(v_{b} \kappa_{4 b, x}+p\right) \prod_{l=1 ; l \neq x}^{u_{b}} \Gamma\left(\kappa_{4 b, l}-\kappa_{4 b, x}\right)}{\kappa_{4 b, x} \prod_{l=1}^{r_{b}} \Gamma\left(\kappa_{3 b, l}-\kappa_{4 b, x}\right)}-\bar{P}_{S C} .
\end{aligned}
$$

In this case, diversity or coding can not be extracted due to the fact that the expression consist of 3 terms and can not be reduced to the form of $\bar{P} \approx\left(G_{c} \cdot \mu\right)^{-G_{d}}$. For the case of Gamma-Gamma, we have

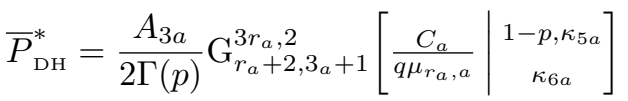

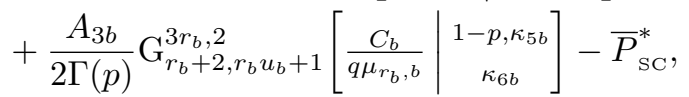

which agrees with [4, Eq. (22)]

3) Ergodic Capacity: Using (34) along with the identities $\ln (1+x)=\mathrm{G}_{2,2}^{1,2}\left[x \mid \begin{array}{l}1,1 \\ 1,0\end{array}\right]$, [50, Eq. (12)], and [46, Eq. (07.34.21.0011.01)], we obtain $\bar{C}_{\mathrm{DH}}$ in closed- form as

$$
\begin{aligned}
& \bar{C}_{\mathrm{DH}}=\frac{A_{3 b}}{\ln (2)(2 \pi)^{v_{b}-1}} \mathrm{G}_{r_{b}+2 v_{b}, u_{b}+2 v_{b}}^{u_{b}+2 v_{b},}\left[\begin{array}{l|l}
\frac{C_{b}}{\left(c \mu_{r_{b}, b}\right)^{v_{b}}} & \kappa_{9 b}, \kappa_{3 b} \\
\kappa_{4 b}, \kappa_{10 b}
\end{array}\right] \\
& +\frac{A_{3 a}}{\ln (2)(2 \pi)^{v_{a}-1}} \mathrm{G}_{r_{a}+2 v_{a}, u_{a}+2 v_{a}}^{u_{a}+2 v_{a}, v_{a}}\left[\begin{array}{c|c}
\frac{C_{a}}{\left(c \mu_{r_{a}, a}\right)^{v a}} & \kappa_{9 a}, \kappa_{3 a} \\
\kappa_{4 a}, \kappa_{10 a}
\end{array}\right] \\
& -\bar{C}_{\mathrm{SC}} \text {, }
\end{aligned}
$$

where $\kappa_{9 i}=\Delta\left(v_{i}, 0\right), \Delta\left(v_{i}, 1\right)$ and $\kappa_{10 i}=\Delta\left(v_{i}, 0\right), \Delta\left(v_{i}, 0\right)$.

For the special case of Gamma-Gamma, we have

$$
\begin{gathered}
\bar{C}_{\mathrm{DH}}^{*}=\frac{A_{3 b}}{\ln (2)} \mathrm{G}_{r_{b}+2,3 r_{b}+2}^{3 r_{b}+2,}\left[\frac{C_{b}}{c \mu_{r_{b}, b}} \mid \begin{array}{l}
0,1, \kappa_{3 b} \\
\kappa_{4 b}, 0,0
\end{array}\right] \\
+\frac{A_{3 a}}{\ln (2)} \mathrm{G}_{r_{a}+2,3 r_{a}+2}^{3 r_{a}+2,1}\left[\frac{C_{a}}{c \mu_{r_{a}, a}} \mid \begin{array}{l}
0,1, \kappa_{3 a} \\
\kappa_{4 a}, 0,0
\end{array}\right]-\bar{C}_{\mathrm{SC}}^{*}
\end{gathered}
$$

in agreement with [4, Eq. (23)].

\section{Two Data Streams Multiplexing}

Assuming two FSO links that transmit independent data to the same destination. The first link requires high data rate 
$D_{1}$ compared to the second FSO link with a lower data rate $D_{2}$. An initial thought is to use the results obtained from the maximum and the minimum in Sections IV-A and IV-B, respectively. The link providing the maximum capacity $\bar{C}_{\mathrm{M}}$ can be identified and $D_{1}$ data rate can be assigned to it. Similarly, the link providing the minimum capacity $\bar{C}_{\mathrm{m}}$ can be identified and $D_{2}$ data rate can be assigned to it. In this work, we try to accommodate each link's need and at the same time try to utilize the network resources efficiently and intelligently. We introduce the parameter $\epsilon \in[0,1]$ which consists of the percentage of time the first data stream is assigned to the channel with the best SNR. Thus, $D_{1}$ and $D_{2}$ can be given as

$$
\begin{aligned}
& D_{1}=\epsilon \bar{C}_{\mathrm{M}}+(1-\epsilon) \bar{C}_{\mathrm{m}}, \\
& D_{2}=(1-\epsilon) \bar{C}_{\mathrm{M}}+\epsilon \bar{C}_{\mathrm{m}} .
\end{aligned}
$$

\section{RESUlts AND Discussion}

In this section, we verify and validate our analytical results of hybrid FSO communication systems, assuming IM/DD $r=$ 2 , using computer-based simulations. We also consider RF link experiencing the same channel fading model.

\section{A. Dual-Branch SC Systems}

First, we investigate the BER performance of dual-branch FSO/FSO $\left(r_{a}=r_{b} \in\{1,2\}\right)$ and RF/FSO $\left(r_{a}=1\right.$ and $\left.r_{b} \in\{1,2\}\right)$ SC systems under differentially coherent binary phase shift keying (DPSK) modulation in which $p=1$ and $q=1$. In this work, we consider two scenarios of atmospheric turbulence conditions, strong (consider the following set of parameters: $\alpha_{1, i}=1.8621, \alpha_{2, i}=1, \beta_{1, i}=0.5, \beta_{2, i}=1.8$, $\Omega_{1, i}=1.5074$, and $\Omega_{2, i}=0.9280$ such that $\lambda_{i}=17$ and $\sigma_{i}=9$ ) and moderate (consider the following set of parameters: $\alpha_{1, i}=2.1690, \alpha_{2, i}=1, \beta_{1, i}=0.55, \beta_{2, i}=2.35$, $\Omega_{1, i}=1.5793$, and $\Omega_{2, i}=0.9671$ where $\lambda_{i}=28$ and $\left.\sigma_{i}=13\right)$.

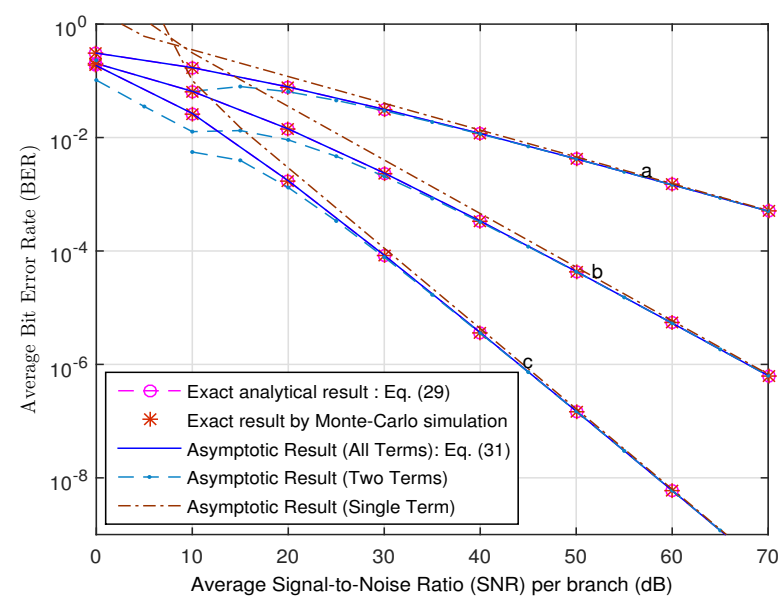

Figure 3: The impact of pointing error $(\xi=1.2)$ on the average BER of DPSK over (a) single FSO link, (b) dual-branch FSO/FSO and (c) RF/FSO $\mathrm{SC}$ in strong turbulence conditions.

First, Fig. 3 shows the impact of pointing errors (i.e strong effect of pointing errors as $\xi \rightarrow 0$ ) on the performance of single FSO link and dual-branch SC of FSO/FSO and RF/FSO. We can notice instantly that SC helps to improve the performance comparing to the single link with expense of utilizing more hardware at the receive side. Also, dual-branch RF/FSO SC performance better than symmetric branches FSO/FSO. This encourages the integration of RF into FSO systems or vice verse. Regarding the asymptotic results, they converge fast to exact ones even for low SNR (i.e starting at $30 \mathrm{~dB}$ ). Interestingly, the asymptotic results representing of all terms in the summation matches perfectly the exact results which give our expression more value instead of using special functions. In the same fashion, two dominants terms asymptotic results provide better match than single dominant term.

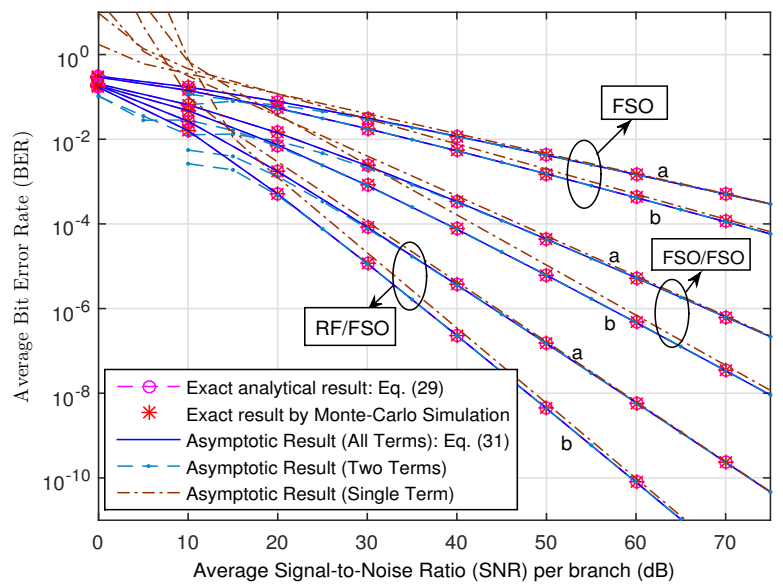

Figure 4: The effect of turbulence conditions ((a) Strong turbulence and (b) Moderate conditions) on the average BER of DPSK over single FSO link, dual-branch FSO/FSO and RF/FSO SC with severe pointing error, $\xi=1.2$

Moreover, Fig. 4 shows the impact of the turbulence conditions on the performance of FSO systems. Clearly, they lead to some degradation. Again, the asymptotic results including all terms in the summation provide an excellent match with the exact result. It is noticeable that for strong conditions the asymptotic expressions including single dominant terms is better than for the moderate conditions and that is due to the fact that the differences between $\left\{\frac{\xi_{i}^{2}}{v_{i} r_{i}}, \frac{\beta_{1 i}}{r_{i} \sigma_{i}}, \frac{\beta_{2 i}}{r_{i} \lambda_{i}}\right\}, i \in\{a, b\}$, for moderate conditions is not significance.

Secondly, we evaluate another performance metric that is the ergodic capacity of dual-branch SC FSO/FSO and RF/FSO $\mathrm{SC}$ as shown in Fig. 5. Generally, SC systems provides higher data rate than a single link system. Also, we can notice that behavior of RF/FSO and FSO/FSO capacity are not in big difference in contrast with BER.

\section{B. Dual-Hop Transmission Systems}

We evaluate BER under DPSK modulation and ergodic capacity of FSO relay systems including dual-hop RF-FSO and FSO-FSO links as shown in Figs. 6 and 7. We had to compare the performance of a FSO link that suffers strong turbulence conditions in addition to severe pointing errors with relay assisted link of RF-FSO and FSO-FSO experiencing moderate turbulence conditions with $\xi>>1$. Clearly, relay links outperform the single link in addition to providing higher 


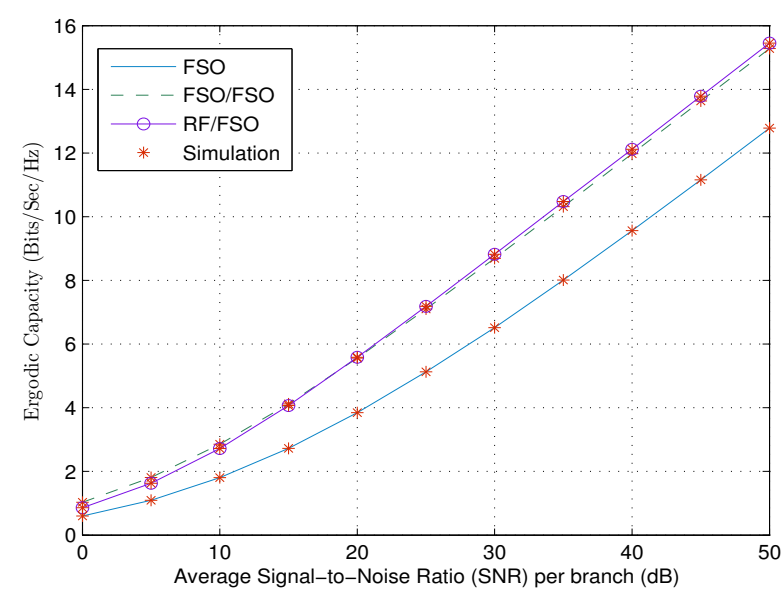

Figure 5: Ergodic capacity of single FSO link, dual-branch FSO/FSO, RF/FSO SC in strong turbulence conditions and under severe pointing errors, $\xi=1.2$.

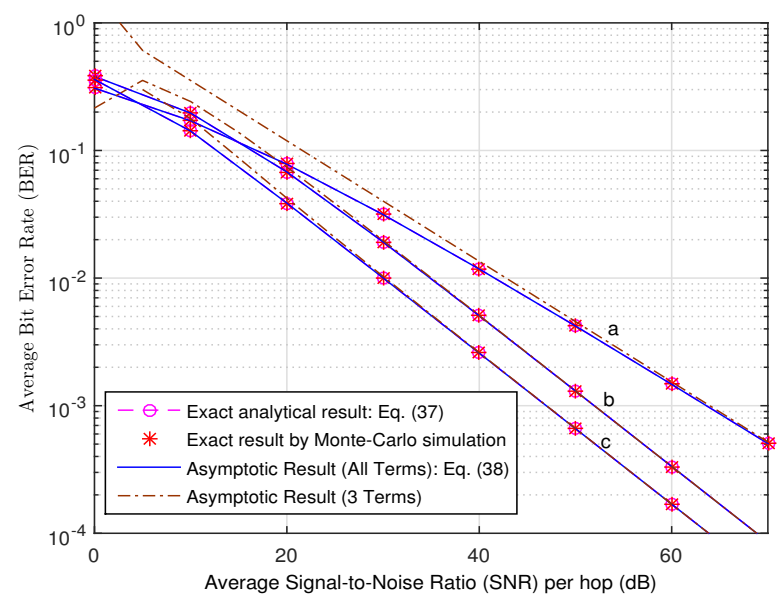

Figure 6: Average BER of DPSK over (a) single FSO link (b) dual-hop FSOFSO (c) RF-FSO

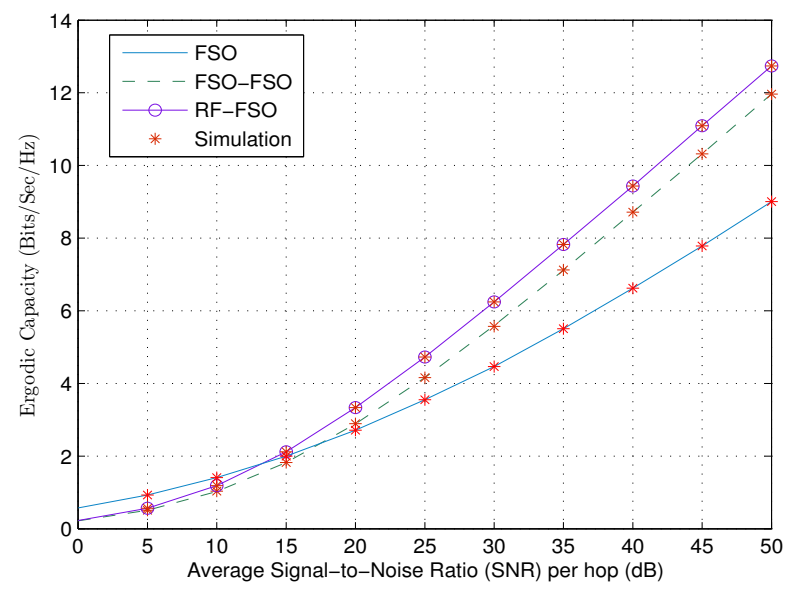

Figure 7: Ergodic capacity of single FSO link and dual-hop FSO-FSO and RF-FSO.

data rate than a single FSO link. Moreover, the asymptotic analysis shows an excellent match with the exact results even for low SNR values (i.e starting at $20 \mathrm{~dB}$ ).

\section{Two Data Streams Multiplexing}

Fig. 8 shows the ergodic capacity of our proposed scheme with different values of $\epsilon$. As observed, as $\epsilon$ increases, $D_{1}$ increases while $D_{2}$ decreases and vice versa. By this model, $D_{1}$ can get data rate that is close to the maximum capacity the system can provide and at the same time $D_{2}$ gets higher data rate than expected.

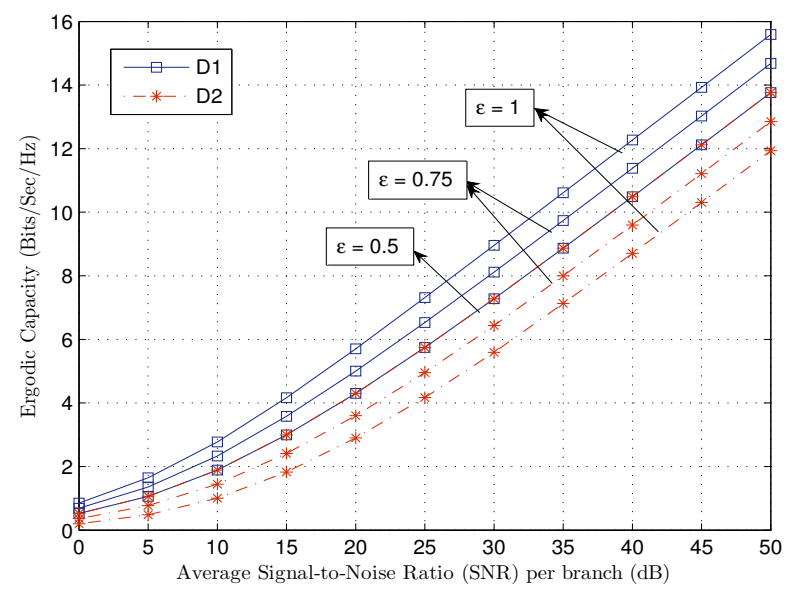

Figure 8: Capacity Multiplexing of two i.i.d FSO link with $\xi=6.5$ in strong turbulence conditions

\section{CONCLUSION}

Closed-form expressions for the CDF, the PDF, the MGF, and the moments of the maximum and the minimum of two modified double generalized gamma variates were derived. Furthermore, we derived analytical expressions for average bit error rate and ergodic capacity and then applied them to find the performance of both dual-branch hybrid FSO SC scheme and dual-hop hybrid FSO relay system. Monte-Carlo computer simulations were carried out to validate our results. Finally, based on our results for the maximum and minimum, we have proposed a data streams multiplexing scheme for two independent FSO links that compromises each link's data rate judiciously in order to utilize the network available capacity in a flexible fashion.

\section{REFERENCES}

[1] M. A. Kashani, M. Uysal, and M. Kavehrad, "A novel statistical channel model for turbulence-induced fading in free-space optical systems," IEEE/OSA J. Lightwave Technol., vol. 33, no. 11, pp. 2303-2312, March 2015.

[2] A. Farid and S. Hranilovic, "Outage capacity optimization for free-space optical links with pointing errors," Journal of Lightwave Technology, vol. 25, no. 7, pp. 1702-1710, July 2007.

[3] H. AlQuwaiee, I. S. Ansari, and M.-S. Alouini, "On the performance of free space optical wireless communication systems over double generalized Gamma fading channel," in Proceedings of 4th IEEE International Conference on Communications and Networking (COMNET' 2014), Hammamet, Tunisia, Mar. 2014

[4] — "On the maximum and minimum of two modified GammaGamma variates with applications," in Proceedings of IEEE Wireless Communications and Networking Conference (WCNC' 2014), Istanbul, Turkey, Apr. 2014, pp. 269-274. 
[5] W. O. Popoola and Z. Ghassemlooy, "BPSK subcarrier intensity modulated free-space optical communications in atmospheric turbulence," IEEE/OSA Journal of Lightwave Technology, vol. 27, no. 8, pp. 967-973, Apr. 2009.

[6] J. Park, E. Lee, and G. Yoon, "Average bit-error rate of the Alamouti scheme in Gamma-Gamma fading channels," IEEE Photonics Technology Letters, vol. 23, no. 4, pp. 269-271, Feb. 2011.

[7] D. Kedar and S. Arnon, "Urban optical wireless communication networks: the main challenges and possible solutions," IEEE Communications Magazine, vol. 42, no. 5, pp. S2-S7, May 2004.

[8] S. Arnon, J. Barry, G. Karagiannidis, R. Schober, and M. Uysal, Advanced Optical Wireless Communication Systems. Cambridge University Press, 2012.

[9] M. Uysal, J. Li, and M. Yu, "Error rate performance analysis of coded free-space optical links over Gamma-Gamma atmospheric turbulence channels," IEEE Transactions on Wireless Communications, vol. 5, no. 6 , pp. 1229-1233, Jun. 2006.

[10] H. Sandalidis, T. Tsiftsis, G. Karagiannidis, and M. Uysal, "BER performance of FSO links over strong atmospheric turbulence channels with pointing errors," IEEE Communications Letters, vol. 12, no. 1, pp. 44-46, Jan. 2008.

[11] H. Sandalidis, T. Tsiftsis, and G. Karagiannidis, "Optical wireless communications with heterodyne detection over turbulence channels with pointing errors," IEEE/OSA Journal of Lightwave Technology, vol. 27, no. 20, pp. 4440-4445, Oct. 2009.

[12] X. Tang, Z. Ghassemlooy, W. O. Popoola, and C. Lee, "Coherent polarization shift keying modulated free space optical links over a Gamma-Gamma turbulence channel," American Journal of Engineering and Applied Sciences, vol. 4, no. 4, pp. 520-530, 2011.

[13] M. K. Simon and M.-S. Alouini, Digital communication over fading channels. New York: Wiley, 2000.

[14] H.-C. Yang, "New results on ordered statistics and analysis of minimumselection generalized selection combining (GSC)," IEEE Transactions on Wireless Communications, vol. 5, no. 7, pp. 1876-1885, July 2006.

[15] Y.-C. Ko, M.-S. Alouini, and M. K. Simon, "Analysis and optimization of switched diversity systems," IEEE Transactions on Vehicular Technology, vol. 49, no. 5, pp. 1813-1831, Sep. 2000

[16] M. Ibrahim and A. Ibrahim, "Performance analysis of optical receivers with space diversity reception," IEEE Transactions on Communications, vol. 134, no. 6, pp. 369-372, Dec. 1996.

[17] E. J. Shin and V. W. Chan, "Optical communication over the turbulent atmospheric channel using spatial diversity," in Proceedings of IEEE Global Telecommunications Conference, 2002 (GLOBECOM'02), vol. 3, Nov. 2002, pp. 2055-2060.

[18] A. Ibrahim, M.M.; Ibrahim, "Part 1: Optical communication over the clear turbulent atmospheric channel using diversity," IEEE Journal on Selected Areas in Communications, vol. 22, no. 9, pp. 1896-1906, Nov. 2004.

[19] H. Moradi, M. Falahpour, H. Refai, and P. LoPresti, "A diversity combining approach for MIMO FSO nodes with misaligned receivers," in Proceedings of Global Telecommunications Conference (GLOBECOM'11), Houston, TX, USA, Apr. 2011, pp. 1-5.

[20] H. Kazemi and M. Uysal, "Performance analysis of MIMO free-space optical communication systems with selection combining," in Proceedings of the 21st Signal Processing and Communications Applications Conference (SIU), Haspolat, Cyprus, April. 2013, pp. 1-4.

[21] T. Tsiftsis, H. Sandalidis, G. Karagiannidis, and M. Uysal, "Optical wireless links with spatial diversity over strong atmospheric turbulence channels," IEEE Transactions on Communications, vol. 8, no. 2, pp. 951-957, Feb. 2009

[22] M. O. Hasna and M.-S. Alouini, "A performance study of dual-hop transmissions with fixed gain relays," in Proceedings of IEEE International Conference on Acoustics, Speech, and Signal Processing (ICASSP '03), vol. 4, Apr. 2003, pp. IV-189-92.

[23] F. Yilmaz, O. Kucur, and M.-S. Alouini, "A novel framework on exact average symbol error probabilities of multihop transmission over amplify-and-forward relay fading channels," in Proceedings of the 7th International Symposium on Wireless Communication Systems (ISWCS), York, UK, Sep. 2010, pp. 546-550.

[24] Y. Zhu, Y. Xin, and P.-Y. Kam, "Outage probability of Rician fading relay channels," in Proceedings of Military Communications Conference (MILCOM), Washington, DC, Sep. 2006, pp. 1-6.

[25] S. N. Datta, S. Chakrabarti, and R. Roy, "Error analysis of noncoherent FSK with variable gain relaying in dual-hop Nakagami-m relay fading channel," in Proceedings of International Conference on Signal Processing and Communications (SPCOM' 2010), Bangalore, India, July 2010, pp. $1-5$.
[26] T. Tsiftsis, H. Sandalidis, G. Karagiannidis, and N. Sagias, "Multihop free-space optical communications over strong turbulence channels," in Proceedings of IEEE International Conference on Communications, (ICC '06), Istanbul, Turkey, June 2006, pp. 2755-2759.

[27] G. Karagiannidis, T. Tsiftsis, and H. Sandalidis, "Outage probability of relayed free space optical communication systems," Electronics Letters, vol. 42, no. 17, pp. 994-995, August 2006.

[28] M. Safari and M. Uysal, "Relay-assisted free-space optical communication," IEEE Transactions on Wireless Communications, vol. 7, no. 12, pp. 5441-5449, December 2008.

[29] E. Lee, J. Park, D. Han, and G. Yoon, "Performance analysis of the asymmetric dual-hop relay transmission with mixed RF/FSO links," IEEE Photonics Technology Letters, vol. 23, no. 21, pp. 1642-1644, Nov. 2011.

[30] I. S. Ansari, M.-S. Alouini, and F. Yilmaz, "On the performance of hybrid RF and RF/FSO fixed gain dual-hop transmission systems," in Proceedings of 2013 Saudi International Electronics, Communications and Photonics Conference (SIECPC), Riyadh, SA, April 2013, pp. 1-6.

[31] H. Samimi and M. Uysal, "End-to-end performance of mixed RF/FSO transmission systems," IEEE/OSA Journal of Optical Communications and Networking, vol. 5, no. 11, pp. 1139-1144, Nov 2013.

[32] S. Ikki and M. Ahmed, "Performance analysis of dual-hop relaying communications over generalized Gamma fading channels," in Global Telecommunications Conference (GLOBECOM '07), Nov. 2007, pp. 3888-3893.

[33] M. Hasna and M.-S. Alouini, "End-to-end performance of transmission systems with relays over Rayleigh-fading channels," IEEE Transactions on Wireless Communications, vol. 2, no. 6, pp. 1126-1131, Nov 2003.

[34] I. Ansari, F. Yilmaz, and M.-S. Alouini, "On the performance of mixed RF/FSO variable gain dual-hop transmission systems with pointing errors," in Proceedings of 78th IEEE Vehicular Technology Conference (VTC Fall), Las Vegas, USA, Sept 2013, pp. 1-5.

[35] M. Aggarwal, P. Garg, and P. Puri, "Dual-hop optical wireless relaying over turbulence channels with pointing error impairments," IEEE/OSA Journal of Lightwave Technology, vol. 32, no. 9, pp. 1821-1828, May 2014.

[36] W. Gappmair, "Further results on the capacity of free-space optical channels in turbulent atmosphere," IET Communications, vol. 5, no. 9, pp. 1262-1267, Jun. 2011.

[37] H. AlQuwaiee, I. S. Ansari, and M.-S. Alouini, "On the performance of free-space optical communication systems over double generalized gamma channel," To appear in IEEE Journal on Selected Areas in Communications - 2014 Special Issue on Optical Wireless Communication.

[38] M. Al-Habash, L. C. Andrews, and R. L. Phillips, "Mathematical model for the irradiance probability density function of a laser beam propagating through turbulent media," Optical Engineering, vol. 40, no. 8, pp. 1554-1562, Feb. 2001

[39] I. S. Ansari, F. Yilmaz, and M.-S. Alouini, "A unified performance of free-space optical links over Gamma-Gamma turbulence channels with pointing errors," in Proceedings of IEEE 81st Vehicular Technology Conference (VTC Spring' 2015), Glasgow, Scotland, May 2015.

[40] - "Impact of pointing errors on the performance of mixed RF/FSO dual-hop transmission systems," IEEE Wireless Communications Letters, vol. 2, no. 3, pp. 351-354, Jun. 2013.

[41] F. Yilmaz and M.-S. Alouini, "Product of the powers of generalized Nakagami-m variates and performance of cascaded fading channels," in Proceedings of IEEE Global Telecommunications Conference, (GLOBECOM'09), Honolulu, USA, Nov 2009, pp. 1-8.

[42] P. Mittal and K. Gupta, "An integral involving generalized function of two variables," Proceedings of the Indian Academy of Sciences Section A, vol. 75, no. 3, pp. 117-123, Mar. 1972

[43] I. S. Ansari, S. Al-Ahmadi, F. Yilmaz, M.-S. Alouini, and H. Yanikomeroglu, "A new formula for the BER of binary modulations with dual-branch selection over generalized- $K$ composite fading channels," IEEE Transactions on Communications, vol. 59, no. 10, pp. 2654-2658, Oct. 2011.

[44] K. Peppas, "A new formula for the average bit error probability of dualhop amplify-and-forward relaying systems over generalized shadowed fading channels," Wireless Communications Letters, IEEE, vol. 1, no. 2, pp. 85-88, April 2012.

[45] K. Gupta and P. Mittal, "The H-function transform II," Journal of the Australian Mathematical Society, vol. 12, no. 04, pp. 444-450, Nov. 1971.

[46] I. Wolfram, Mathematica Edition: Version 8.0. Champaign, Illinois: Wolfram Research Inc., 2010. 
[47] Z. Wang and G. Giannakis, "A simple and general parameterization quantifying performance in fading channels," IEEE Transactions on Communications, vol. 51, no. 8, pp. 1389-1398, Aug 2003.

[48] A. Lapidoth, S. Moser, and M. Wigger, "On the capacity of free-space optical intensity channels," IEEE Transactions on Information Theory, vol. 55, no. 10, pp. 4449-4461, Oct 2009.

[49] S. Arnon, J. Barry, G. Karagiannidis, R. Schober, and M. Uysal, Advanced optical wireless communication systems. Cambridge University Press, 2012.

[50] S. Gupta, "Integrals involving products of G-function," Proceedings of the National Academy of Sciences, India Section A: Physical Sciences, vol. 39, no. 2, pp. 193-200, 1969.

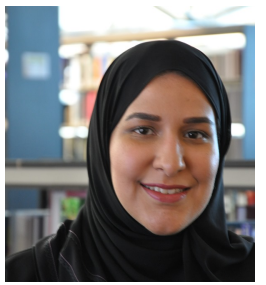

Hessa AlQuwaiee (S'09) received the B.S degree in Computer Engineering from Prince Mohamed University (PMU), Alkhobar, Saudi Arabia in 2011. In 2008, she was awarded the discovery scholarship to complete graduate studies in King Abdullah University of Science and Technology (KAUST). In 2013, she earned her M.S. degree in Electrical Engineering. She is now toward her $\mathrm{PhD}$ degree at KAUST. Her current research interests include, but not limited to, channel characterization and performance analysis of optical wireless communications, body-to-body communications, diversity combining techniques and MIMO systems.

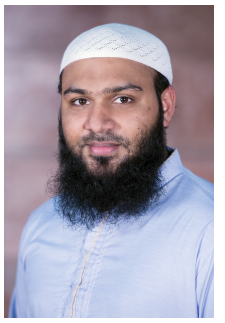

Imran Shafique Ansari (S'07, M'15) was born in India in 1987. He completed his B.Sc. in Computer Engineering from King Fahd University of Petroleum and Minerals (KFUPM) in 2009 (with First Honors) and M.Sc. and PhD from King Abdullah University of Science and Technology (KAUST) in 2010 and 2015, respectively. Currently, he is a Postdoctoral Research Associate (PRA) with Texas A\&M University at Qatar (TAMUQ). From May 2009 through Aug. 2009, he was a visiting scholar with Michigan State University (MSU), East lansing, MI, USA, and from Jun. 2010 through Aug. 2010, he was a research intern with Carleton University, Ottawa, ON, Canada. He has been a student member of IEEE and IET since 2007 and has served in various capacities. He has served on IET CC-EMEA (Communities Committee-Europe, Middle-East and Africa) for a complete term from Oct. 2010-Sep. 2013 and has been re-elected to serve for another term from Oct. 2015-Sep. 2018. He is an active reviewer for various IEEE Transactions and various other journals. He has served as a TPC for various IEEE conferences. He is a recipient of recognized reviewer certificate by Elsevier Optics Communications in 2015, a recipient of recognized reviewer certificate by OSA Publishing in 2014 a recipient of appreciation for an exemplary reviewer for IEEE Wireless Communications Letters (WCL) in 2014, a recipient of post-doctoral research award (PDRA) (first cycle) with Qatar national research foundation (QNRF) in 2014, a recipient of KAUST academic excellence award (AEA) in 2014, and a recipient of IEEE Richard E. Merwin student scholarship award in Jul. 2013. Imran has authored/co-authored around 34 journal and conference publications. His current research interests include free-space optics (FSO), channel modeling/signal propagation issues, relay/multihop communications, physical layer secrecy issues, and diversity reception techniques among others.

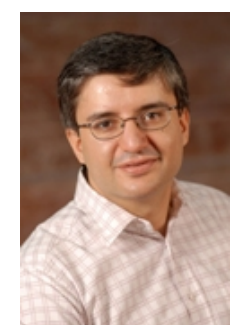

Mohamed-Slim Alouini (S'94, M'98, SM'03, F'09) was born in Tunis, Tunisia. He received the Ph.D. degree in Electrical Engineering from the California Institute of Technology (Caltech), Pasadena, CA, USA, in 1998. He served as a faculty member in the University of Minnesota, Minneapolis, MN, USA, then in the Texas A\&M University at Qatar, Education City, Doha, Qatar before joining King Abdullah University of Science and Technology (KAUST), Thuwal, Makkah Province, Saudi Arabia as a Professor of Electrical Engineering in 2009. His current research interests include the modeling, design, and performance analysis of wireless communication systems. 